\title{
Variations in Gleason grading during active surveillance
}

The issue of Gleason upgrading after diagnostic biopsy is well-documented, but poses a particular concern for men on active surveillance, where an increase in grade can trigger radical intervention. Two recent studies have addressed Gleason score in men being managed expectantly; the first characterized grade changes over an extended period of surveillance, and the second uncovered a specific issue in the interpretation of Gleason patterns 3 and 4 on follow-up biopsy.

Sima Porten and colleagues at the University of California, San Francisco examined the changes in Gleason score over 8 serial biopsies (mean follow-up of 54 months) in 377 men on active surveillance. Researchers found that upgrading occurred less frequently with each subsequent biopsy. Overall, 129 patients (34\%) experienced an upgrade, $81 \%$ of whom were upgraded on their first or second repeat biopsy. Given the long natural history of prostate cancer, the authors suggest that men who experience early upgrading are victims of initial sampling error, whereas those whose biopsies reveal an upgrade after a longer interval are likely to be experiencing true disease progression.

The second study, performed by Jesse McKenney and team in multiple centers across North America, identified a problem in distinguishing between the poorly-formed glands of Gleason 4 disease and tangentially-sectioned Gleason 3 tissue.

Investigators constructed three sets of images from prostate needle core biopsies, and asked 11 pathologists to assign Gleason scores to each. Set 1 comprised a selection of cases representing classic Gleason patterns 3, 4 and 5, one of which caused significant disagreement between reviewers. While six pathologists believed a focal area of small glands to be

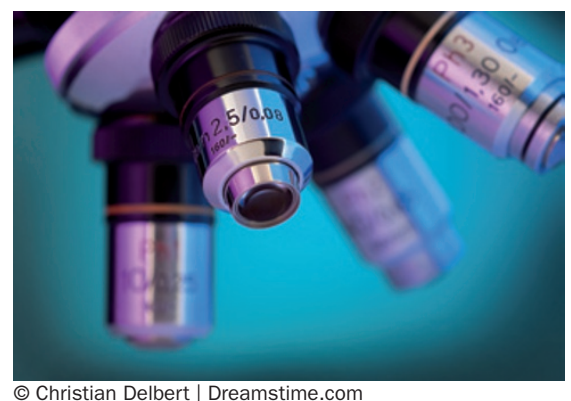

tangentially-sectioned Gleason pattern 3, five others assigned a Gleason score of 4 .

To address this discrepancy further, set 2 was compiled of images of the ambiguous pattern. Using Light's $\kappa$ analysis, researchers found that interobserver reproducibility was considerably lower for set 2 than set $1(\kappa=0.27$ versus $\kappa=0.75)$.

Finally, researchers used image set 3-which consisted of the same images as set 2, but in a different order and shown to reviewers a month later-to assess intraobserver reproducibility, yielding values of $65-100 \%$. Pathologists with a lower diagnostic threshold for assigning Gleason 4 demonstrated better reproducibility.

The Gleason 3/4 boundary is especially important in this setting, given that Gleason pattern 4 on follow-up biopsy is a trigger for intervention in most active surveillance programs. The authors suggest that separating Gleason pattern 4 into cribriform and poorly-formed subtypes might help in the future.

\section{Sarah Payton}

Original articles Porten, S. P. et al. Changes in prostate cancer grade on serial biopsy in men undergoing active surveillance. J. Clin. Oncol. doi:10.1200/ JC0.2010.33.0134 | McKenney, J. K. et al. The potential impact of reproducibility of Gleason grading in men with early stage prostate cancer managed by active surveillance: a multi-institutional study. J. Urol. doi:10.1016/ j.juro.2011.03.115 